\title{
A Simple Method for Optic Disk Segmentation from Retinal Fundus Image
}

\author{
Adithya Kusuma Whardana \\ Departement of Informatic, Faculty of Information Technology, Institut Teknologi Sepuluh November, Surabaya, \\ Indonesia \\ Email: blue_kenshi@hotmail.com \\ Nanik Suciati \\ Departement of Informatic, Faculty of Information Technology, Institut Teknologi Sepuluh November, Surabaya, \\ Indonesia \\ Email: nanik@if.its.ac.id
}

\begin{abstract}
Detection of optic disc area is complex because it is located in an area that is considered as pathological blood vessels when in segmentation and thus require a method to detect the area of the optic disc, this paper proposed the optic disc segmentation using a method that has not been used before, and this method is very simple, K-means clustering is a proposed Method in this paper to detect the optic disc area with perfected using adaptive morphology. This paper successfully detect optic disc area quickly and segmented blood vessels more quickly.
\end{abstract}

Index Terms - Optic Disk segmentation, Blood Vessel, $\mathrm{K}$-means, adaptive morphology.

\section{INTRODUCTION}

Blood vessels in the retina is part of the retina that serves to supply blood and oxygen to the blood vessel of the retina. If the blood and oxygen supplies are not smooth, then this may be a reference to detect whether there are health problems (hypertension, cardiovascular, stroke or diabetes). To detect these problematic veins that can be done with the segmentation of blood vessels in retinal digital images. There have been many approaches made to handle segmentation of blood vessels (supervised and un-supervised), but still do not meet the problems of state-of-the-art [1]: (1) The existence of the reflection of light from the center, (2) blood vessel, (3) Branching blood vessels, (4) Merging of adjacent blood vessels, (5) Loss of blood vessels are thin / small and (6) Error detection of blood vessels in the area.

The retina is a highly organized structure with the ability to start the processing of visual information before the information is transmitted through the optic nerve to the visual cortex. Layered structure that allows the function to search restrictions in optical disk function or functional impairment on a layer or group of cells. However, the perception of color, contrast, depth, and shape take place in the cortex [8].

Aliaa Abdel-Haleim Abdel-Razik Youssif et al [2] Optic Disc (OD) is one of the main features of fundus images of the retina (Fig. 1.) is an original image taken from the dataset VDIS. OD is one of the most important from indicator opthamology pathology. There was some research done for the optic disc segmentation but before segmentation must be clearly for detected boundary area from centroid for OD previous research can be done with active contour model [14]. There are several steps described in previous studies [2], initially mask binary image produced on the retina, then the illumination equalization to locate the OD is shown in Fig. 2. [2], the next step adaptive histogram equalization receipts to detect small areas of blood vessels.

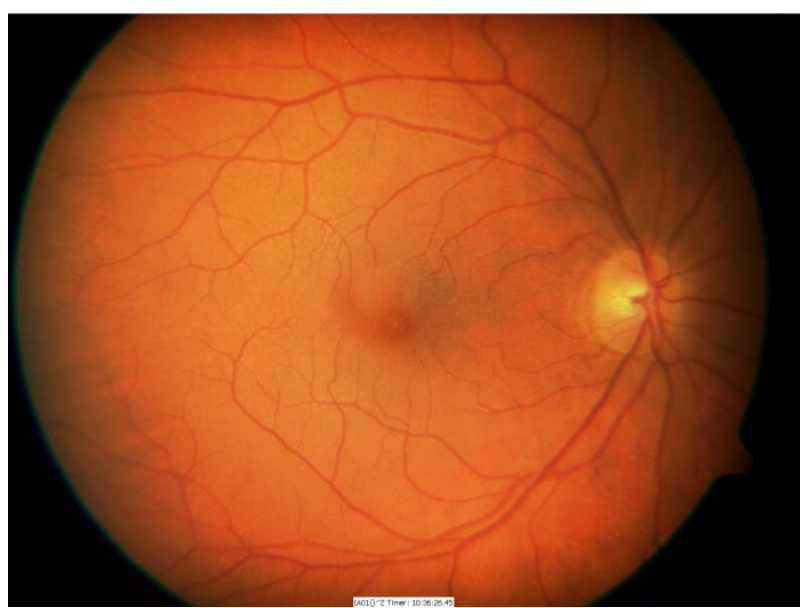

Fig 1. Original Image retinal fundus image.

Welfer et al [3-4] Segmentation is necessary to separate the optic disc area with blood vessels that exist in the area of the optic disc at the time was not considered in the segmentation of blood vessels.

This paper proposed the Combaining of two methods, $\mathrm{K}$-means clustering method to determine the area of the optic disc to be in the segmentation in retinal fundus images, which in previous studies has not been used for the detection of optic disc area, optic disc area after extraction to separate the optic disc area the area of the blood vessels so the next step using adaptive morphology [3-4]. By using the K-means faster detection, in previous 
studies [2]. Dataset used for the test data and training data using the DRIVE database. The results of the evaluation and segmentation process is done by using the mathlab 2013 summarized and presented.

The structure of this paper is given as: section 2 describes the proposed method and sequence of steps in the research that has been done. Finally, the paper is concluded in section 3 .

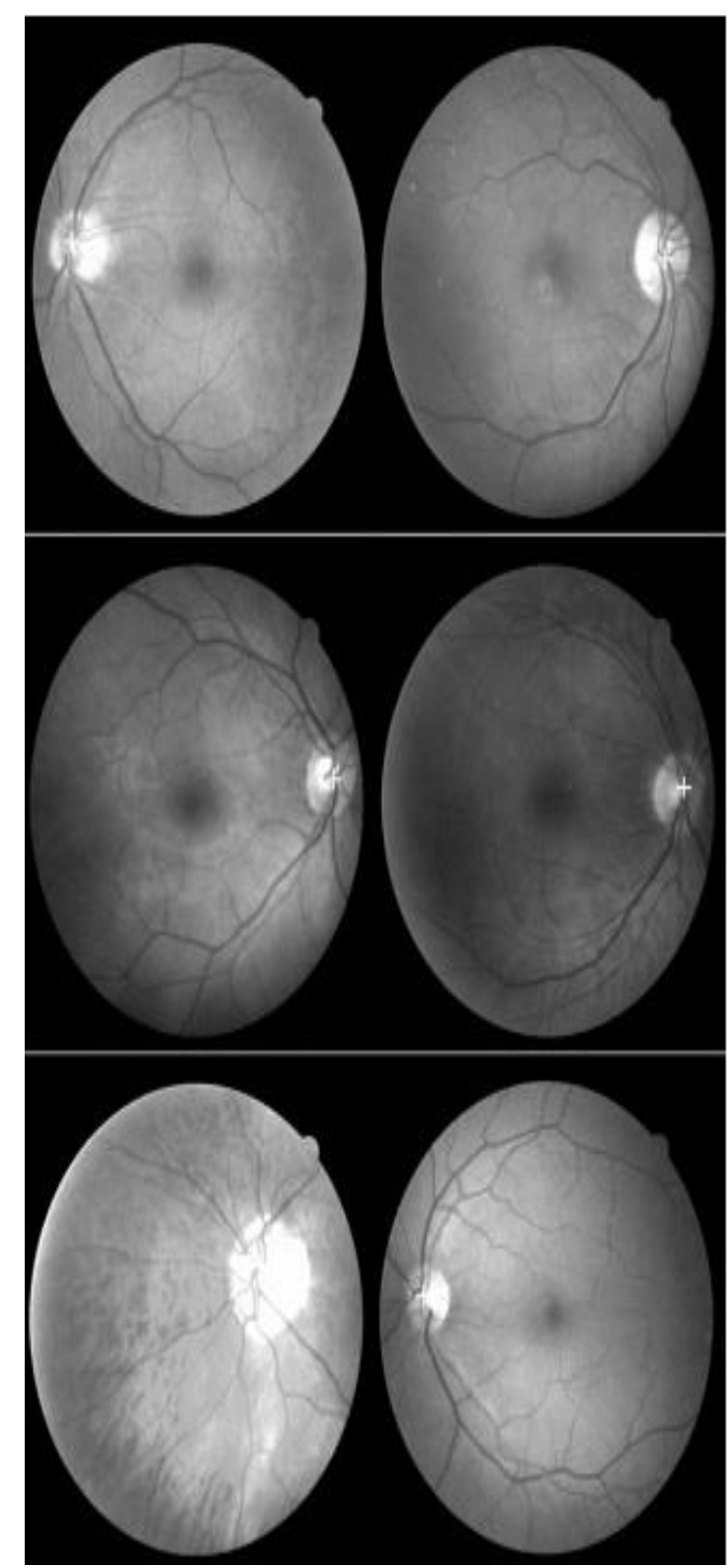

Fig 2. Shown the related work using the DRIVE dataset white cross represents the estimated OD center [2].

\section{PROPOSED METHOD}

\section{A. Preprocessing}

In a previous study [2] optic disc is one of the main keys of the retina, because of the difficult position in determining the area because the area is very close to the blood vessels, so that the optical disk intersect with the blood vessels, the optic disc becomes difficult in the segmentation process because the area is very difficult to be detected in the OD localization of the optic disc area using thresholding [6]. A lot of the optic disk detection process that has been done in previous research. As has been throughly by[9], the process of using the watershed transform preprocessing optic disc area then detected by using internal and external markers marker.

Rashid Jalal Qureshi et al [10] optic disk detection is essential in the optic disc segmentation process, because it is a major component in opthamological pathology which is an indicator of the eye [20], the Topological Active net one [17] of the many methods that use the calculation of the intensity of the color is less successful as well as by using active contour model [18] so it took a few methods to be successful in finding the optic disc that signals the optic disc area, this paper explains to sign on area optical disks need only need the one way by using the K-means .

This paper proposed a method that has never been used before for optic disc area detected Fig.3 shows the proposed method in the detection of optic disc.

$$
\begin{gathered}
\text { input image } \rightarrow \text { Green Channel } \rightarrow \text { Optic Disk Detection } \\
\text { K-Means }
\end{gathered}
$$

Fig 3. Flow Diagram Proposed Method.

In the first step of preprocessing, input the original image will be on segmentation, the original image to the image of the fox and the green channel image of the green channel reshaping the image of the function reshape the K-means clustering method, after the reimage forming green channel the next step in the algorithms K-means clustering mentioned that the total value of $k$ is assumed as a cluster. The main idea is to determine the centroid $\mathrm{k}$, where $k$ is the area of the optic disc to be in the detection. The mathematics' expression of this method(1)[7].

$$
\mathrm{E}=\sum_{j=1}^{k} \sum_{i_{1} \in c_{j}}\left|i_{1}-w_{j}\right|^{2}
$$

K-Means method is used to detect the optical disk area that will in the following segmentation of the sequence of steps in the flow diagram of the proposed method show in fig. 4. To detect the optic disc area given the choice of which areas will be detected later in the segmentation area already, If want to detect the optic disc area should be chosen first new area next to the area of the optical disc automatically be detected and then be in segmentation using Adaptif morphological methods that have been done earlier researchers [3-4]. This paper proposed a new adaptive method with a faster than previous researcher so that blood vessels can be segmented area clearly. 

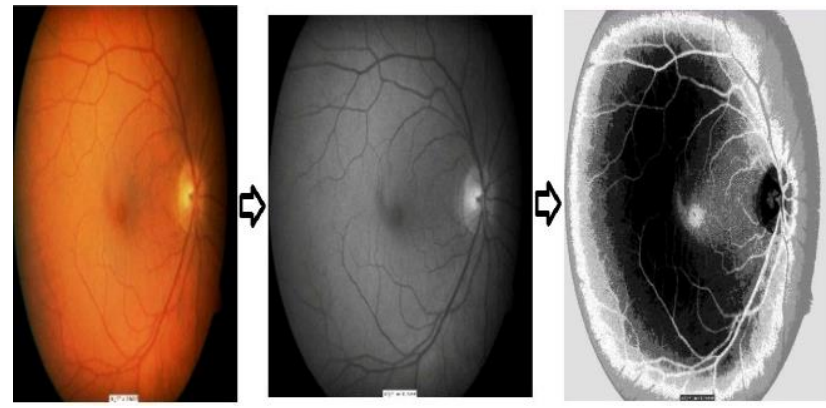

Fig. 4. A.image original. B. Green Channel image. C.Reshape image.

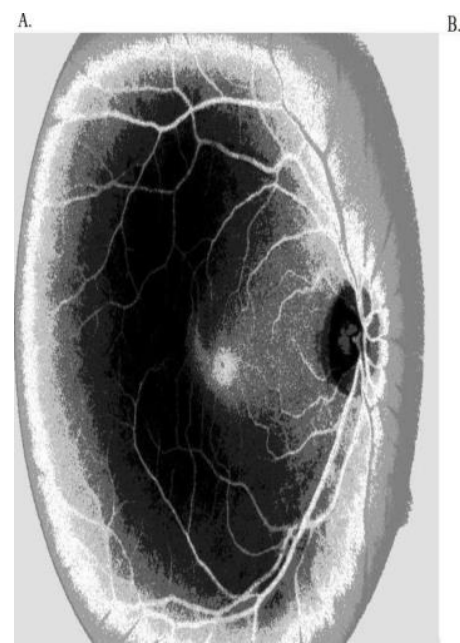

Fig 5. A. Rhesape of image that of the K-means clustering method. B.Optic disk area selected during the process of reshaping the image.

\section{B. Optic Disk Detection}

Optic disc in healthy retina marked by bright circular area on the optic disc [11], so that when the detection area is easily detected by using entropy map [12]. In that area could also be detected by using curvelet transform [13]. In the region of interest (Roi) can be extracted using the average pixel [14] and take the picture with the red channel gives the area centroid as the center [15].

Carla Agurto et al [16] using topological active nets, capable of segmenting the image of the optic disc by integrating features of the region segmentation technique and edge areas.

In the previous picture image reshape Fig.4c. is an implementation of the existing $\mathrm{K}$-means clustering method [7], after that it is in the detection of optic disc should then select the area in which one will be detected is shown in Fig. 5, Fig. 5. is an image that will reshape the detection of optic disc area in retinal fundus images, small black circle area close to the area of pathological blood vessels is an area that will be in the optic disk detection.

\section{Optic Segmentation}

Once the optic disc area in detection by using the proposed method uses the K-means clustering Pseudo

code of the proposed method showing in Fig. 6. The next step, segmentation of optic disc area using Adaptive morphology that has been done in related work [3]. In this paper, after the area detected the optical disk is assumed as centroid then the step is much faster than step on the previous researchers.

$$
\begin{aligned}
& \mathrm{idx}=\operatorname{kmeans}(\mathrm{rgb}, \mathrm{k}) \\
& \mathrm{hasil}=\operatorname{reshape}(\mathrm{idx}, \mathrm{m}, \mathrm{n}) ; \\
& \mathrm{hasil}=(\text { hasil-1)/(k-1); }
\end{aligned}
$$

Fig 6. PseudoCode proposed Method.

\section{Vessel Segmentation}

Jan,J et all [19] the process of blood vessel segmentation using directional 2D matched filtering and estimation with Neural Fibre layer of high-resolution images taken from the camera. segmentation of blood vessels in retinal image had been in previous thorough and succeeded in segmenting using the line detection [5].

Where is detected during the next step of segmentation optic disc is in the area of adaptive morphology pathological using, Fig.7. is the steps in related work [3]. In the previous steps described there are several stages, the first stage, the RGB image to the image of the fox green channel, then the image in the image of the marker by using the function RMIN operator then for some later step using the operator skeleton image, the last image in the pruning step.
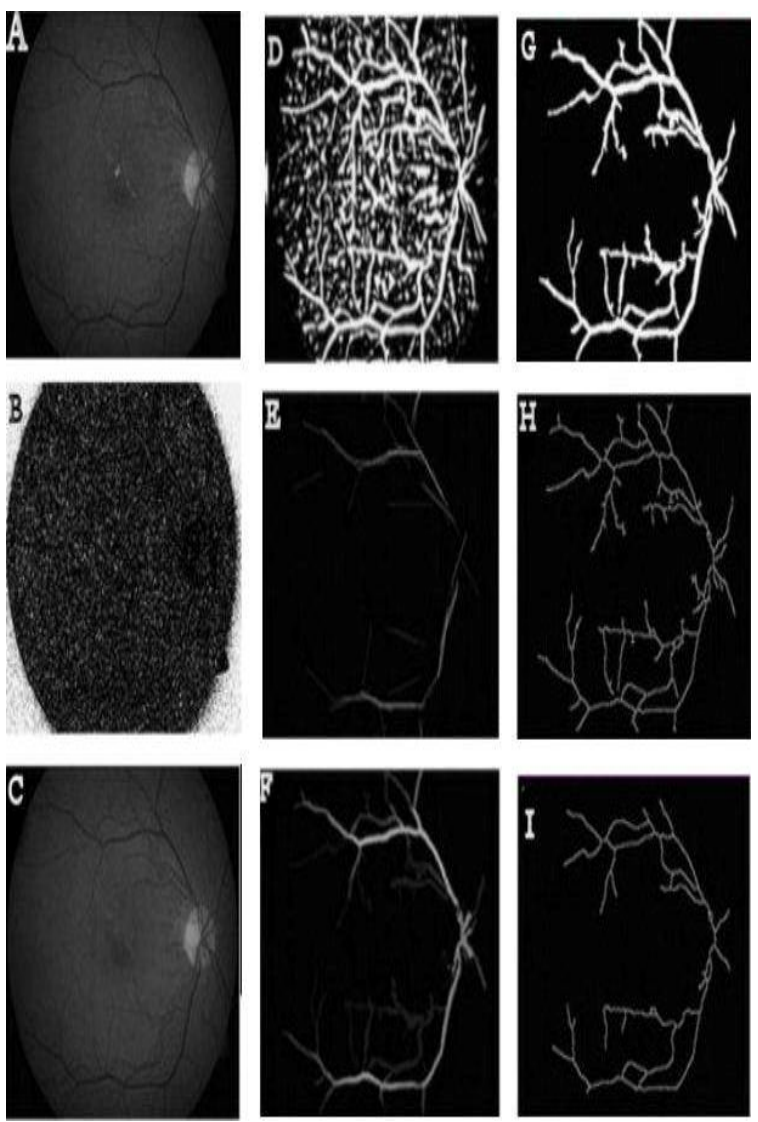

Fig 7. Related work adaptive morphology [3].

Morphological method is one method of image segmentation to make improvements on the retina, 
resulting in retinal images could be well segmented and clearly [3-4]. Process morphology such as spatial convolution in a pixel value based on the value of neighboring pixels to construct a new vessel of the array and the array pixel mask [4].

Daniel welfer et al [3] adaptive morphological method used morphological method is one method of image segmentation to make improvements on the retina, resulting in retinal images can be segmented properly and clearly. Morphological operations are common operations imposed on the binary image (black and white) to change the structure of the object shape is contained in the image[21].Morphological image processing is usually done on a binary image only consisting of 0 and 1 do not rule out the possibility of the image with gray scale $0-255$ That will be studied is the binary image morphological processing In general, morphological image processing is done by mem-passing a structuring element to an image in a manner similar to the convolution.

The basic principle is the use of adaptive morphological structure element (SE) that form the basis of an object that is used to analyze the geometry of other objects larger and more complex. The goal obtain the information from a form of image segmentation would be particularly here in the retinal image, adjust the shape of the retina and the retinal size so that what you want in segmentation can be an important information[21].

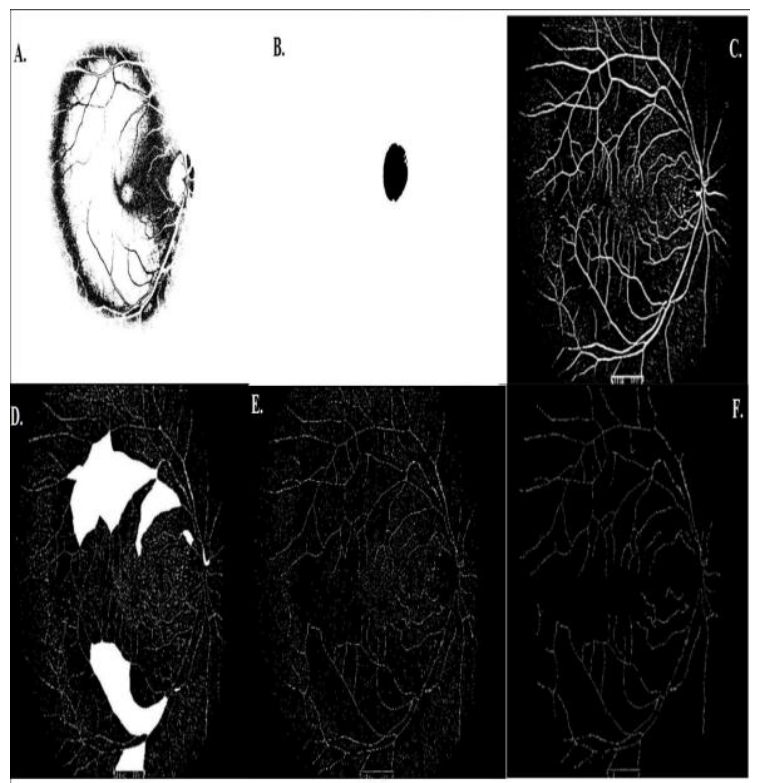

Fig 8. A. Feature extraction. B. area Optic disk C. image segmentation result before going on the segmentation of optic disc area. D. hole image E. area in the optic disk segmentation. F. blood vessel segmented with optic disk segmented.

The following steps segmentation of blood vessels using an adaptive step adaptive morphology [3]. Researchers propose new adaptive morphology method with simple steps and faster than previous research, Fig. 8 is a segmentation step of the optic disc and blood vessels.

In fig. $8 \mathrm{~A}$ is the result of feature extraction using the $\mathrm{K}$ means algorithm, after selecting which areas will be in segmentation, for optic disc area in the area of No. 4 or 5 .
In Fig.8B, an area that will be in the optic disc segmentation, Fig.8C is an image segmentation result before going on the segmentation of optic disc area, Fig. $8 \mathrm{D}$ image is determining which areas will be in the blood vessels segmentation. Fig. 8E. area in the optic disk segmentation, image segmentation Fig.8F. is the end result.

\section{Hole Image (fh)}

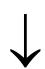

Skeleton Image (fs)

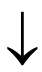

\section{Spur Morphology image (fsi)}

Fig 9. Proposed Method Blood Vessel.

Step in the segmentation of blood vessels in the fig. 9 . This paper proposed that where a faster method than previous researchers [3-4] By using adaptive morphology operators.

In Fig. 8B, an area that will be in the optic disc

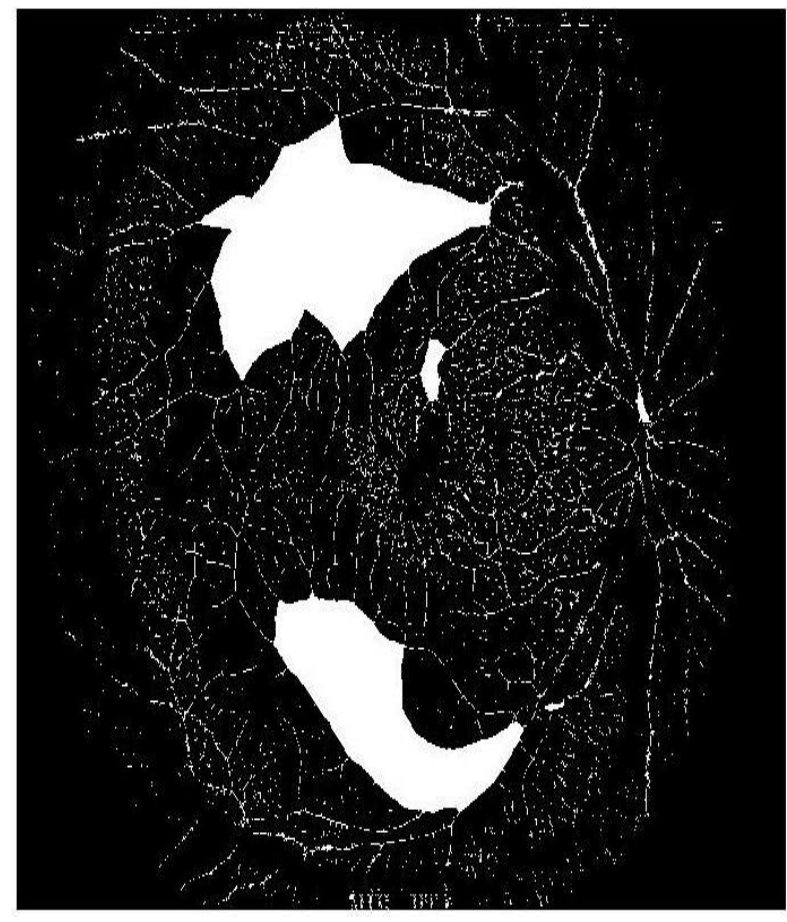

Fig 10. Hole image (fh)

segmentation, Fig.8C is an image segmentation result before going on the segmentation of optic disc area, Fig. $8 \mathrm{D}$ image is determining which areas will be in the blood vessels segmentation. Fig. 8E. area in the optic disk segmentation, image segmentation Fig.8F is the end result. 


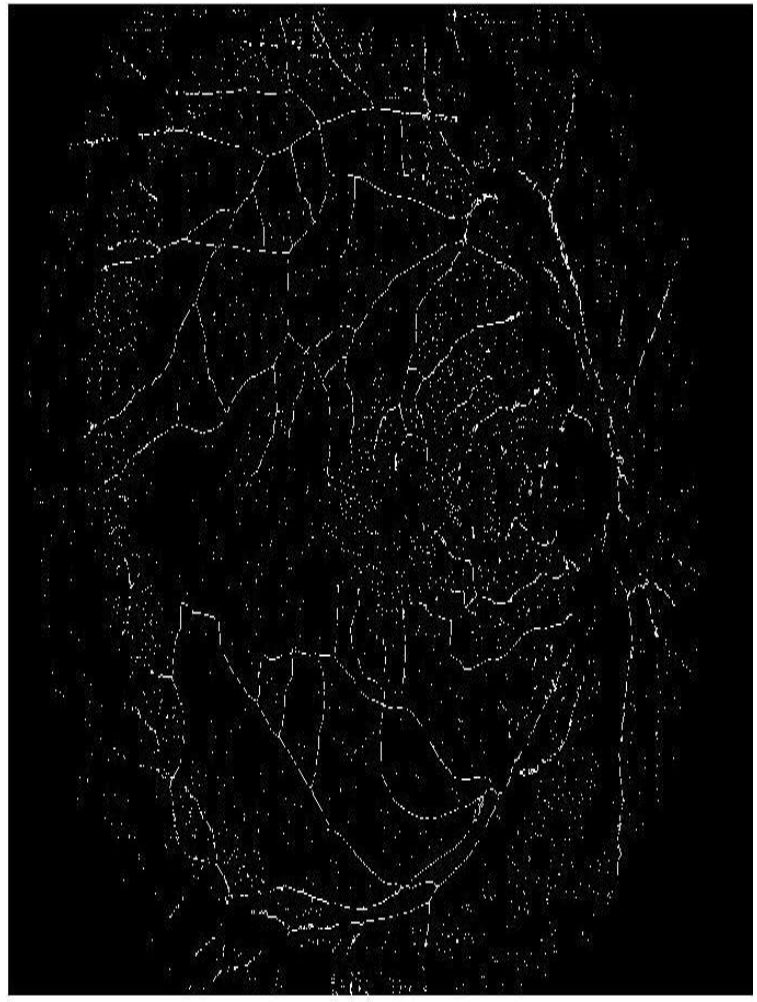

Fig 11. Skeleton Image (fs).

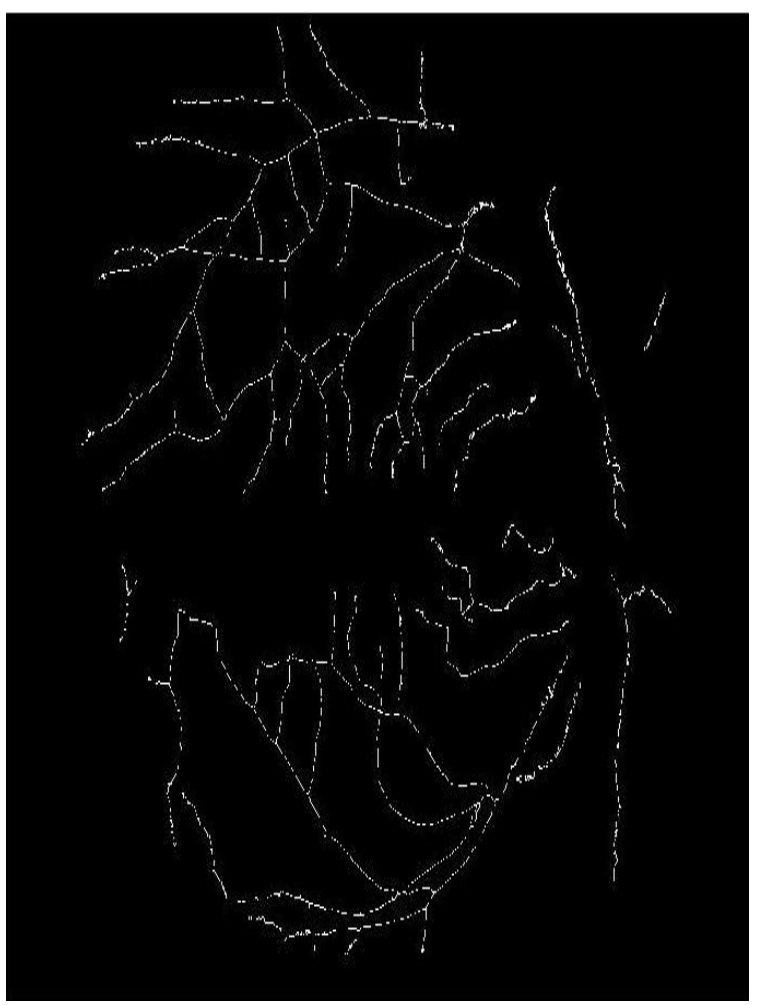

Fig 12. Spur Morphology Image (fsi).

The first step in the segmentation process by using the hole in the morphology operators, assuming $(f h)$ where the image after the process of looking for a hole in the detection of optic disc, the optic disc area has been separated by an area of blood vessels that are in the area of pathological blood vessels segmentation process can be well. The next step after the next process in the $f h$ then did the skeleton image is assumed $(f s)$, fs is a function of the existing morphology operators in mathlab, the next step in image Morphology spur assume $(f s i)$. Fig. 10. is an image of $f h$.

Hole function Morphological operations area common operations imposed on the binary image (black and white) to change the structure of the object shape is contained in the image[3-4]. Fig. 11. is an image of $f s$. Skeleton morphology is another way of reducing the object of the binary image into several blood vessels become thin [3]. Fig. 12 is an image of $f$ si. Spur is a function operating on adaptive morphology, which is useful for removing pixels spur in the retinal image. Fig. 13 is an image of the Pseudocode operating $f$ si.

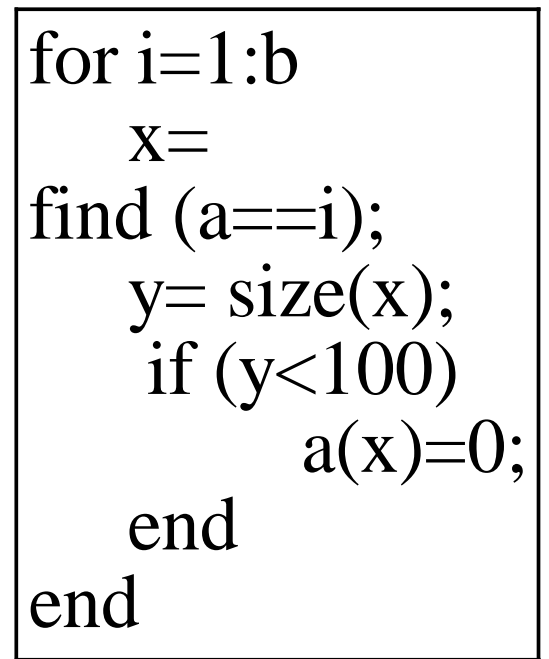

Fig 13.Pseudocode operating fsi.

The algorithms of the training phase and the testing

phase of blood vessel are given below The mathematics' expression of this algorithm is showing (2).

$$
\begin{aligned}
& f h=\text { holes image } \\
& f s=\text { skeleton image }=(100 \text { pixel }) \\
& f s i=\text { Spuring image } \\
& \text { if } \mathrm{y}<100 \\
& \text { else } \\
& x=0
\end{aligned}
$$

The (2) is equation for segmented for blood vessel after the optic disk segmented exactly. $f h$ is an image that is in the hole after the detection of the optical disc by using $\mathrm{K}$ means clustering method, after the area was taken from the optic disc area of new blood vessel segmentation can. fh binary image with holes filled, then after the next process filled holes using adaptive morphology operation in the skeleton skeleton operators are already available in mathlab, $f s$ is skeletonize images of $f h$ that later after the $f s$ process spuring image is done by searching for the value of adjacency between each where $f s$ blood vessels in the estimate of 100 pixels when the value of the 
neighboorhood is less than 100 then the area is not the area of the blood vessel will then blacked out.

\section{CONCLUSION}

In this work, this paper proposed a simple method for segmenting optic disc area using K-means clustering and adaptive morphology. In this paper to detect the optic disc area using the k-means clustering method, and then to segment the optic disk and blood vessel morphology using an adaptive method. This paper proposed a new method and has not been used in the segmentation of optic disc and with the incorporation of the methods that have been used by previous researchers [3-4] This method produces good segmentation, so that the area of the optic disc in retinal blood vessels are not detected as not truncated, and the area of the blood vessels more clearly on the ground truth area. In this test successfully implement a retinal image better than previous researchers, by means of a faster step than before researchers. in this work that using the k-means clustering method to detect optic disc area and using adaptive morphological method there is the disadvantage that cutting some blood vessels and lack of clarity in the area of the blood vessel, and there are several veins are truncated when segmentation. in the execution of this paper uses a database of DRIVE and implementation of methods using Mathlab. Researchers propose to repair the blood vessels by using multi-scale line detection.

\section{ACKNOWLEDGMENT}

Researcher thank to the ITS laboratory has Provided the research facility under construction. and DRIVE databases and Mathlab who have helped in the operation method.

\section{REFERENCES}

[1] Nguyen, Uyen TV, Alauddin Bhuiyan, Laurence AF Park, and Kotagiri Ramamohanarao. "An effective retinal blood vessel segmentation method using multi-scale line detection." Pattern Recognition (2012).

[2] Youssif, Aliaa Abdel-Haleim Abdel-Razik, Atef Zaki Ghalwash, and Amr Ahmed Sabry Abdel-Rahman Ghoneim. "Optic Disc Detection From Normalized Digital Fundus Images by Means of a Vessels' Direction Matched Filter." IEEE TRANSACTIONS ON MEDICAL IMAGING 27.1 (2008): 11.

[3] Welfer, Daniel, Jacob Scharcanski, Cleyson M. Kitamura, Melissa M. Dal Pizzol, Laura WB Ludwig, and Diane Ruschel Marinho. "Segmentation of the optic disk in color eye fundus images using an adaptive morphological approach." Computers in Biology and Medicine 40 (2010):124-137.

[4] Welfer, Daniel, Jacob Scharcanski, and Diane Ruschel Marinho. "A morphologic two-stage approach for automated optic disk detection in color eye fundus images." Pattern Recognition Letters (2012).

[5] Wihandika, Randy Cahya, and Nanik Suciati. "Retinal Blood Vessel Segmentation with Optic Disc Pixels Exclusion." International Journal of Image, Graphics \& Signal Processing 5.7 (2013).
[6] Kaur, Jaspreet, and H. P. Sinha. "Automated Localisation of Optic Disc and Macula from Fundus Images." International Journal 2.4 (2012).

[7] Alsabti, Khaled, Sanjay Ranka, and Vineet Singh. "An efficient k-means clustering algorithm." (1997).

[8] Riordan-Eva, P., \& Whitcher, J. (Eds.). (2008).Vaughan \& Asbury's general ophthalmology. Lange Medical Books/McGraw-Hill Medical Pub. Division.

[9] Eswaran, C., Reza, A. W., \& Hati, S. (2008, August). Extraction of the Contours of Optic Disc and Exudates Based on Marker-Controlled Watershed Segmentation.In International Conference on Computer Science and Information Technology 2008 (pp. 719-723). IEEE.

[10] Qureshi, R. J., Kovacs, L., Harangi, B., Nagy, B., Peto, T., \& Hajdu, A. (2012). Combining algorithms for automatic detection of optic disc and macula in fundus images. Computer Vision and Image Understanding, 116(1), 138145.

[11] Ramakanth, S. A., \& Babu, R. V. (2014). Approximate Nearest Neighbour Field based Optic Disk Detection. Computerized Medical Imaging and Graphics, 38(1), 4956.

[12] Mendonça, A. M., Sousa, A., Mendonça, L., \& Campilho, A. (2013). Automatic localization of the optic disc by combining vascular and intensity information. Computerized Medical Imaging and Graphics.

[13] Esmaeili, M., Rabbani, H., \& Dehnavi, A. M. (2012). Automatic optic disk boundary extraction by the use of curvelet transform and deformable variational level set model. Pattern Recognition, 45(7), 2832-2842.

[14] Muramatsu, C., Nakagawa, T., Sawada, A., Hatanaka, Y., Hara, T., Yamamoto, T., \& Fujita, H. (2011). Automated segmentation of optic disc region on retinal fundus photographs: Comparison of contour modeling and pixel classification methods. Computer methods and programs in biomedicine, 101(1), 23-32.

[15] Kumar, V., \& Sinha, N. (2013, April). Automatic Optic Disc segmentation using maximum intensity variation. In TENCON Spring Conference, 2013 IEEE (pp. 29-33). IEEE.

[16] Agurto, C., Yu, H., Murray, V., Pattichis, M. S., Barriga, S., Bauman, W., \& Soliz, P. (2012, August). Detection of neovascularization in the optic disc using an AM-FM representation, granulometry, and vessel segmentation. In Engineering in Medicine and Biology Society (EMBC), 2012 Annual International Conference of the IEEE (pp. 4946-4949). IEEE.

[17] Novo, J., Penedo, M. G., \& Santos, J. (2009). Localisation of the optic disc by means of GA-optimised Topological Active Nets. Image and Vision Computing, 27(10), 15721584.

[18] Zheng, Y., Stambolian, D., O’Brien, J., \& Gee, J. C. (2013). Optic Disc and Cup Segmentation from Color Fundus Photograph Using Graph Cut with Priors. In Medical Image Computing and Computer-Assisted Intervention-MICCAI 2013 (pp. 75-82). Springer Berlin Heidelberg.

[19] Jan, J., Odstrcilik, J., Gazarek, J., \& Kolar, R. (2012). Retinal image analysis aimed at blood vessel tree segmentation and early detection of neural-layer deterioration. Computerized Medical Imaging and Graphics, 36(6), 431-441.

[20] Xu, J., Sung, E., Chutatape, O., Zheng, C., \& Chew, P. (2006, December). Automated optic disk segmentation via a modified snake technique. In Control, Automation, Robotics and Vision, 2006. ICARCV'06. 9th International Conference on (pp. 1-6). IEEE. 
[21] Gonzalez, Rafael C., Richard E. Woods, and Steven L. Eddins. Digital image processing using MATLAB. Vol. 2. Tennessee: Gatesmark Publishing, 2009.

\section{Authors' Profiles}

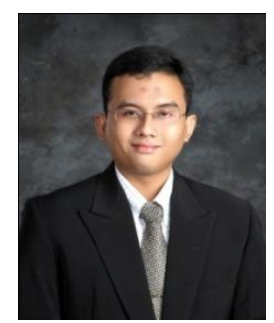

Adithya Kusuma Whardana, Male, received S.Kom degree from Information System Universitas Pembangunan Nasional "Veteran" East Java, Surabaya, in 2008 . He is currently a master student at Department of Informatics, Institut Teknologi Sepuluh Nopember, Surabaya, Indonesia. His research interests include image processing, pattern recognation, Computer Vision, mobile computing.

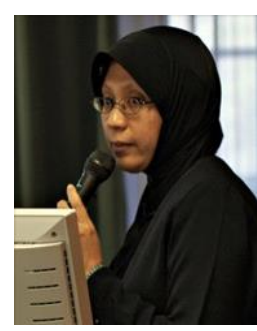

Nanik Suciati, Female, received ph.D. degree from Hiroshima University, Japan in 2010 in information Engginering. She is Currently an academic member of the department of informatics, The Faculty of information Technology, Institut Teknologi Sepuluh November, Surabaya, Indonesia. Her research interests include image processing, computer vision, computer graphics, and data mining.

How to cite this paper: Adithya Kusuma Whardana, Nanik Suciati,"A Simple Method for Optic Disk Segmentation from Retinal Fundus Image", IJIGSP, vol.6, no.11, pp.36-42, 2014.DOI: 10.5815/ijigsp.2014.11.05 